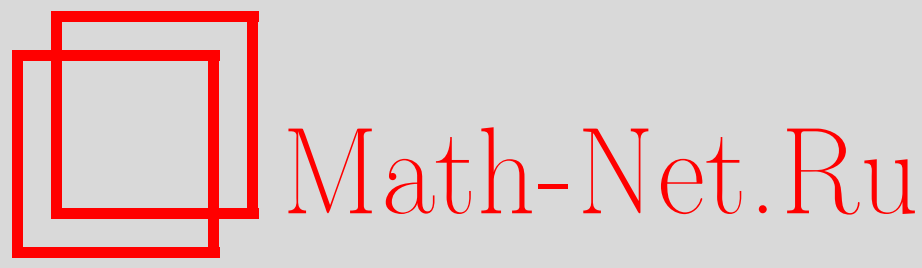

А. И. Зенчук, Решения многомерных уравнений в частных производных, представимых в виде одномерного потока, ТМФ, 2014, том 178, номер 3, 346-362

DOI: https://doi.org/10.4213/tmf8597

Использование Общероссийского математического портала Math-Net.Ru подразумевает, что вы прочитали и согласны с пользовательским соглашением http://www.mathnet.ru/rus/agreement

Параметры загрузки:

IP : 52.90 .164 .192

26 апреля 2023 г., 13:49:42

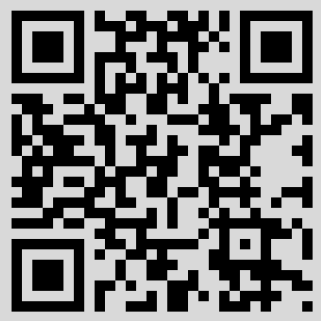




\title{
ФИЗИКА
}

Том 178, № 3

март, 2014

(C) 2014 г.

А. И. Зенчук*

\section{РЕШЕНИЯ МНОГОМЕРНЫХ УРАВНЕНИЙ В ЧАСТНЫХ ПРОИЗВОДНЫХ, ПРЕДСТАВИМЫХ В ВИДЕ ОДНОМЕРНОГО ПОТОКА}

\begin{abstract}
Предложен алгоритм, с помощью которого $(M+1)$-мерное нелинейное уравнение в частных производных, представимое в виде одномерного потока, $u_{t}+w_{x_{1}}\left(u, u_{x}, u_{x x}, \ldots\right)=0$ (где $w$ - произвольная локальная функция от $u$ и ее производных по $\left.x_{i}, i=1, \ldots, M\right)$, сводится к семейству $M$-мерных нелинейных уравнений в частных производных $F(u, w)=0$, где $F$ - общее (или частное) решение некоторого двумерного нелинейного уравнения в частных производных второго порядка. В частности, $M$-мерное уравнение может оказаться обыкновенным дифференциальным уравнением, которое в некоторых случаях может быть проинтегрировано с получением явных решений исходного $(M+1)$-мерного уравнения. Кроме того, можно ввести спектральный параметр в функцию $F$, что приводит к линейному спектральному уравнению, связанному с исходным уравнением. Представлены простейшие примеры нелинейных уравнений в частных производных вместе с их явными решениями.
\end{abstract}

Ключевые слова: метод характеристик, теория интегрируемости, граничные условия, частные решения, редукции низшей размерности.

DOI: $10.4231 / \operatorname{tmf} 8597$

\section{1. ВВЕДЕНИЕ}

Хорошо известно, что метод характеристик [1] позволяет интегрировать уравнения в частных производных первого порядка произвольной размерности. Этот метод оказался полезным и при изучении $(1+1)$-мерных систем гидродинамического типа, для которых была развита модификация этого метода (обобщенный метод годографа) [2]-[5]. Однако введение производных более высокого порядка в нелинейное уравнение либо разрушает интегрируемость, либо требуется другой метод интегрирования. Например, $(1+1)$-мерное уравнение Бюргерса без вязкости представляет собой простейшее нелинейное уравнение, полностью интегрируемое методом характеристик:

$$
u_{t}-u u_{x}=0 .
$$

${ }^{*}$ Институт проблем химической физики РАН, Черноголовка, Московская обл., Россия. E-mail: zenchuk@itp.ac.ru 
Добавляя производную второго порядка в это уравнение, получим хорошо известное $(1+1)$-мерное уравнение Бюргерса с вязкостью:

$$
u_{t}+u_{x x}-u u_{x}=0 .
$$

Это уравнение также полностью интегрируемо, хотя требует применения другого подхода. А именно, оно линеаризуется подстановкой Хопфа-Кола [6]-[8]. Однако уравнение Бюргерса с вязкостью более высокой размерности не может быть полностью проинтегрировано с помощью линеаризации, так что для его исследования необходим другой подход. Например, его решения, разрушающиеся за конечный промежуток времени, изучались методом ренормализационных групп в работах [9], [10]. Далее, вводя производную третьего порядка в уравнение (1), получим уравнение Кортевега-де Фриза (КдФ)

$$
u_{t}+u_{x x x}-u u_{x}=0,
$$

интегрируемое методом обратной задачи рассеяния (МОЗР) [11]-[16], который является еще одним плодотворным методом интегрирования большого класса нелинейных уравнений в частных производных.

В работе [17] показано, что, деформируя характеристику $(1+1)$-мерного уравнения (1), можно получить семейство частных решений для большого класса $(M+1)$ мерных нелинейных эволюционных уравнений высокого порядка с нелинейностью типа нелинейности уравнения КдФ, которое описывается $M$ - или $(M-1)$-мерным нелинейным уравнением. В частности, существует класс $(2+1)$-мерных уравнений в частных производных, решения которых удовлетворяют соответствующим обыкновенным дифференциальным уравнениям (ОДУ), которые, в свою очередь, могут иметь явные решения.

В настоящей работе мы обобщаем алгоритм, предложенный в статье [17], на уравнения, представимые в виде одномерного потока:

$$
u_{t}+w_{x_{1}}\left(u, u_{x}, u_{x x}, \ldots\right)=0,
$$

где $w$ - произвольная локальная функция $u$ и ее производных по $x_{i}, i=1, \ldots, M$, $M$ - число переменных $x_{i}$ в списке $x=\left\{x_{1}, x_{2}, \ldots, x_{M}\right\}$. Мы покажем, что это уравнение обладает большим многообразием решений, описываемых уравнением $F(u, w)=0$ (или $F\left(x_{1}+u t, w\right)=0$ ), где $F$ удовлетворяет некоторому двумерному уравнению в частных производных второго порядка. Это уравнение справедливо для любого нелинейного $(M+1)$-мерного уравнения, представимого в виде (4). Мы приведем простейшие примеры уравнений вместе с их решениями.

Статья имеет следующую структуру. В разделе 2 рассматривается нелинейное уравнение (4) с $w=-u^{2} / 2+f\left(u_{x}, u_{x x}, \ldots\right)$, частные решения которого зависят от характеристики уравнения (1). Уравнение (4) с произвольным $w$ рассматривается в разделе 3. В этом случае решения уравнения не зависят от характеристики уравнения (1). Уравнение в частных производных второго порядка для функции $F$ обсуждается в разделе 4. Спектральное уравнение для нелинейного уравнения (4) получено в разделе 5 , где также обсуждается полнота пространства решений. Частные случаи уравнения $F=0$, имеющего явные решения, представлены в разделе 6 . Выводы приводятся в разделе 7. 
2. УРАВНЕНИЕ $u_{t}-u u_{x_{1}}+f_{x_{1}}\left(u_{x}, u_{x x}, \ldots\right)=0$

В этом разделе предложен алгоритм построения семейства $M$-мерных редукций для нелинейного уравнения, представимого в виде (4) с функцией $w$, имеющей частный вид

$$
w=-\frac{1}{2} u^{2}+f\left(u_{x}, u_{x x}, \ldots\right),
$$

что обобщает уравнения, рассмотренные в работе [17]. Здесь $f$ - произвольная функция от производных $u$ любого порядка по $x_{i}, i=1, \ldots, M$, но не зависящая от $u$. Заметим, что полную частную производную $w_{x_{1}}$ можно представить в виде

$$
w_{x_{1}}=Q u_{x_{1}},
$$

где $Q$ - дифференциальный оператор

$$
Q=-u+\sum_{i=1}^{M} f_{u_{x_{i}}} \partial_{x_{i}}+\sum_{i_{1}, i_{2}=1}^{M} f_{u_{x_{i_{1}} x_{i_{2}}}} \partial_{x_{i_{1}} x_{i_{2}}}+\cdots
$$

Это позволяет представить уравнение (4) в виде

$$
E(u)=u_{t}+Q u_{x_{1}}=0 .
$$

Важно, что функция $w$ удовлетворяет линейному уравнению

$$
E(w)=w_{t}+Q w_{x_{1}}=0,
$$

которое можно вывести непосредственно. В дальнейшем нам понадобится следующее очевидное соотношение между $E(u)$ и $E(w)$ :

$$
E(w)=Q E(u),
$$

которое можно вывести, применив оператор $Q$ к уравнению (8) и используя соотношение (6) вместе с $Q u_{t}=w_{t}$. Семейство $M$-мерных редукций уравнения (4) с $w$ в виде (5) строится с помощью следующей теоремы.

Теорема 1. Пусть функиия и является решением нелинейного $M$-мерного уравнения

$$
F(\xi, w)=0, \quad \xi=x_{1}+t u,
$$

где функиия $F(\xi, w)$ удовлетворяет двумерному уравнению второго порядка

$$
F_{\xi \xi}+F_{w w} \frac{F_{\xi}^{2}}{F_{w}^{2}}-2 F_{\xi w} \frac{F_{\xi}}{F_{w}}=M(F, \xi, w), \quad \xi=t u+x_{1},\left.\quad F_{w}\right|_{F=0} \neq 0 .
$$

Здесь $M$ - некоторая функиия от $F, \xi, w$, удовлетворяющая условию

$$
\left.M(F, \xi, w)\right|_{F=0}=0 .
$$

Тогда функиия $\psi$,

$$
\psi \equiv E(u)=u_{t}+w_{x_{1}},
$$


является решением линейного уравнения

$$
\left(F_{\xi} t+F_{w} Q\right) \psi=0
$$

Если, кроме того, функиия $\psi$ удовлетворяет нулевым граничным условиям, то бункция и является решением нелинейного уравнения (4) с w вида (5).

ДокАЗАтЕльство. Чтобы вывести нелинейное уравнение (4) из уравнения (11), мы, прежде всего, продифференцируем уравнение (11) по $x_{i}$ и $t$ :

$$
\begin{aligned}
E_{x_{1}} & :=F_{\xi}\left(1+t u_{x_{1}}\right)+F_{w} w_{x_{1}}=0, \\
E_{x_{i}} & :=F_{\xi}\left(t u_{x_{i}}\right)+F_{w} w_{x_{i}}=0, \quad i>1, \\
E_{t} & :=F_{\xi}\left(u+t u_{t}\right)+F_{w} w_{t}=0 .
\end{aligned}
$$

Если $\left.F_{\xi}\right|_{F=0}=\left.F_{w}\right|_{F=0}=0$, то уравнения (16)-(18) становятся тождествами. Предположим, что $\left.F_{w}\right|_{F=0} \neq 0$ (случай $\left.F_{\xi}\right|_{F=0} \neq 0$ может быть рассмотрен аналогичным образом). Тогда, решая уравнения (16) и (17) относительно $w_{x_{1}}$ и $w_{x_{i}}(i>1)$ соответственно, получим:

$$
w_{x_{i}}=-\frac{F_{\xi}}{F_{w}}\left(\delta_{1 i}+t u_{x_{i}}\right), \quad i \geqslant 1
$$

Рассмотрим вторые производные уравнения (11) по произвольным парам $x_{i}, x_{j}$ и подставим выражение (19) для производных $w_{x_{i}}, i \geqslant 1$ :

$$
F_{\xi} t u_{x_{i} x_{j}}+F_{w} w_{x_{i} x_{j}}+\left(\delta_{1 i}+t u_{x_{i}}\right)\left(\delta_{1 j}+t u_{x_{j}}\right)\left(F_{\xi \xi}+F_{w w} \frac{F_{\xi}^{2}}{F_{w}^{2}}-2 F_{\xi w} \frac{F_{\xi}}{F_{w}}\right)=0 .
$$

Если $F$ удовлетворяет нелинейному уравнению $(12)$, то последний член в уравнении (20) исчезает, в результате уравнение (20) сводится к виду

$$
F_{\xi} t u_{x_{i} x_{j}}+F_{w} w_{x_{i} x_{j}}=0
$$

Решая это уравнение относительно $w_{x_{i} x_{j}}$, получим связь между вторыми производными функций $w$ и $u$ :

$$
w_{x_{i} x_{j}}=-\frac{F_{\xi}}{F_{w}} t u_{x_{i} x_{j}}
$$

По индукции получим для производной любого порядка $D=\prod_{i=1}^{M} \partial_{x_{i}}^{n_{i}}$ (где $n_{i}-$ произвольные целые числа) следующее соотношение:

$$
F_{\xi} t D u+F_{w} D w=0 .
$$

Следовательно, для дифференциального оператора $\widetilde{Q}=Q+u$ (с оператором $Q$ вида (7)) можно написать

$$
E_{\widetilde{Q}}:=F_{\xi} t \widetilde{Q} u_{x_{1}}+F_{w} \widetilde{Q} w_{x_{1}}=0 .
$$

Теперь рассмотрим следующую комбинацию уравнений (16), (18), (24):

$$
E_{t}-u E_{x_{1}}+E_{\widetilde{Q}}=0
$$


имеющую вид

$$
F_{\xi} t E(u)+F_{w} E(w)=0 .
$$

В силу соотношения (10) можно переписать уравнение (26) в виде уравнения (15) с функцией $\psi$ вида (14). Если $\psi$ удовлетворяет нулевым граничным условиям, то $\psi \equiv E(u) \equiv 0$, что эквивалентно уравнению (4) с функцией $w$, заданной выражением (5). Теорема доказана.

Теперь следует сделать несколько замечаний.

1. Область определения переменных $x_{i}, i=1, \ldots, M$, может быть как ограниченной, так и неограниченной.

2. Для частного выбора функции $F$,

$$
F(\xi, w)=\xi+w
$$

уравнение (11) преобразуется к виду, рассмотренному в работе [17].

3. Если функция $u$ является решением системы $(4),(5)$ и $(11)$, причем $\left.F_{w}\right|_{F=0} \neq 0$, то она удовлетворяет уравнению

$$
u_{t}-\frac{F_{\xi}}{F_{w}}\left(1+t u_{x_{1}}\right)=0 .
$$

В частности, если функция $F$ берется в виде (27), то уравнение $(28)$ принимает вид

$$
u_{t}=t u_{x_{1}}+1
$$

полученный в работе [17]. Для вывода уравнения (28) нужно продифференцировать уравнение (11) по $x_{1}$, решить полученное уравнение относительно $w_{x_{1}}$ и подставить в уравнение (4).

4. Поскольку дифференциальный оператор $Q(7)$ зависит от функции $f$ в выражении (5) для функции $w$, нулевые граничные условия, налагаемые на функцию $\psi$ (14), полностью определяются вышеупомянутой функцией $f$ и не зависят от частного вида функции $F(\xi, w)$.

ПримеР 1. Рассмотрим в качестве примера нелинейное уравнение

$$
u_{t}+u_{x_{1} x_{2}}-u u_{x_{1}}+\alpha\left(u_{x_{2}}^{2}\right)_{x_{1}}=0
$$

которое можно считать деформацией уравнения

$$
u_{t}+u_{x_{1} x_{2}}-u u_{x_{1}}=0
$$

рассмотренного в работе [17]. В этом случае

$$
w=u_{x_{2}}-\frac{u^{2}}{2}+\alpha u_{x_{2}}^{2}
$$

Используя выражение (27) в качестве простого решения уравнения (12) с $M=0$, перепишем уравнение (11) в виде

$$
u_{x_{2}}+\alpha u_{x_{2}}^{2}=\frac{u^{2}}{2}-t u-x_{1} .
$$


Решая это уравнение относительно $u_{x_{2}}$, получим

$$
u_{x_{2}}=-\frac{1}{2 \alpha}\left(1 \pm \sqrt{1+2 \alpha\left(u^{2}-2\left(t u+x_{1}\right)\right)}\right) .
$$

Интегрирование этого ОДУ приводит к функции $u$ в следующем неявном виде:

$$
h_{ \pm}\left(u, x_{1}, t\right)=C\left(x_{1}, t\right)+x_{2},
$$

где

$$
\begin{aligned}
h_{ \pm}\left(u, x_{1}, t\right)= & \frac{1}{2 \sqrt{\eta}}\left(\ln \frac{\sqrt{\eta}-u+t}{\sqrt{\eta}+u-t} \pm \ln \frac{\sqrt{\eta}-u+t}{\sqrt{\eta}+u-t} \pm\right. \\
& \left. \pm \ln \frac{2 \alpha(\eta+\sqrt{\eta}(u-t))-\sqrt{2 \alpha(u-t)^{2}-2 \alpha \eta+1}-1}{2 \alpha(\eta-\sqrt{\eta}(u-t))-\sqrt{2 \alpha(u-t)^{2}-2 \alpha \eta+1}-1}\right) \pm \\
& \pm \sqrt{2 \alpha} \ln \left(\sqrt{2 \alpha}(u-t)+\sqrt{2 \alpha(u-t)^{2}-2 \alpha \eta+1}\right),
\end{aligned}
$$

при этом

$$
\eta=t^{2}+2 x_{1}
$$

Здесь функция $C$ не может быть произвольной, поскольку должна удовлетворять нулевому граничному условию для функции $\psi$,

$$
\psi=u_{t}+u_{x_{1} x_{2}}-u u_{x_{1}}+\alpha\left(u_{x_{2}}^{2}\right)_{x_{1}},
$$

рассматриваемой в качестве решения уравнения (15) с $F_{\xi}=F_{w}=1$ и одномерным дифференциальным оператором первого порядка $Q=\left(\left(1+2 \alpha u_{x_{2}}\right) \partial_{x_{2}}-u\right)$. Таким образом, нам нужно одно граничное условие в точке, например $x_{2}=0$ :

$$
\left.\psi\right|_{x_{2}=0}=0, \quad \text { при этом }\left.u\right|_{x_{2}=0}=\chi\left(x_{1}, t\right) .
$$

Записав уравнение $(29)$ в точке $x_{2}=0$, получим

$$
\chi_{t}=t \chi_{x_{1}}+1
$$

Таким образом,

$$
\chi=A(\eta)+t
$$

Подставляя $\chi$ из (41) в $(35),(36)$ с $x_{2}=0$, приходим к выводу, что $C$ является функцией только от $\eta$. Это, в свою очередь, позволяет считать $C(\eta)$ произвольной функцией $\eta$ в уравнении (35), а граничное условие (39) следует считать неявным определением функции $A(\eta)$ через функцию $C(\eta)$ :

$$
\begin{aligned}
C(\eta)= & h_{ \pm}\left(A(\eta)+t, x_{1}, t\right) \Rightarrow \\
C(\eta)= & \frac{1}{2 \sqrt{\eta}}\left(\ln \frac{\sqrt{\eta}-A(\eta)}{\sqrt{\eta}+A(\eta)} \pm \ln \frac{\sqrt{\eta}-A(\eta)}{\sqrt{\eta}+A(\eta)} \pm\right. \\
& \left. \pm \ln \frac{2 \alpha(\eta+\sqrt{\eta} A(\eta))-\sqrt{2 \alpha A^{2}(\eta)-2 \alpha \eta+1}-1}{2 \alpha(\eta-\sqrt{\eta} A(\eta))-\sqrt{2 \alpha A^{2}(\eta)-2 \alpha \eta+1}-1}\right) \pm \\
& \pm \sqrt{2 \alpha} \ln \left(\sqrt{2 \alpha} A(\eta)+\sqrt{2 \alpha A^{2}(\eta)-2 \alpha \eta+1}\right) .
\end{aligned}
$$


Если $\alpha \ll 1$, то разложение выражения (36) по степеням $\alpha$ (до линейного по $\alpha$ члена) имеет вид

$$
\begin{aligned}
& h_{+}=\frac{1}{\sqrt{\eta}} \ln \frac{\sqrt{\eta}-u+t}{\sqrt{\eta}+u-t}+\sqrt{2 \alpha} \ln 2+\alpha(u-t)+o(\alpha), \\
& h_{-}=-\alpha(u-t)-\sqrt{2 \alpha} \ln 2+o(\alpha) .
\end{aligned}
$$

Таким образом, решение $u$, соответствующее $h_{-}$в уравнении $(35)$, становится сингулярным при $\alpha \rightarrow 0$. Решение $u$, соответствующее $h_{+}$, можно представить в виде

$$
u=u_{1}+\frac{\alpha}{2}\left(C_{2}(\eta)-u_{1}\right)\left(u_{1}^{2}-\eta\right)+o(\alpha),
$$

где $u_{1}$ - решение уравнения (31), полученное в работе [17]:

$$
u_{1}=t+\sqrt{\eta} \frac{1+\widehat{C}(\eta) e^{x_{2} \sqrt{\eta}}}{1-\widehat{C}(\eta) e^{x_{2} \sqrt{\eta}}}, \quad \widehat{C}(\eta)=e^{\left(C_{1}(\eta)-\sqrt{2 \alpha} \ln 2\right) \sqrt{\eta}},
$$

а произвольная функция $C(\eta)$ в выражении (35) представлена в виде $C(\eta)=C_{1}(\eta)+$ $\alpha C_{2}(\eta)$. Рассматривая решения с конечными асимптотиками при $t \rightarrow \infty$, заметим, что $u_{1} \rightarrow 0$ при $t \rightarrow \infty$. Таким образом, если $C_{2}(\eta)=\widetilde{C}_{2}(\eta) / \eta$ и $\widetilde{C}_{2}(\eta) \rightarrow c_{2}=$ const при $t \rightarrow \infty$, то $u \rightarrow-\alpha c_{2} / 2$ при $t \rightarrow \infty$. Если же $C_{2}(\eta) \rightarrow 0$ при $t \rightarrow \infty$, то $u \rightarrow 0$ (вплоть до $о(\alpha))$.

\section{3. УРАВНЕНИЕ (4) С ПРОИЗВОЛЬНОЙ ФУНКЦИЕЙ $w$}

Теперь изменим алгоритм, изложенный в разделе 2 , с целью построения решений уравнения (4) более общего вида, в котором $w$ - произвольная функция от $u$ и ее производных по $x_{i}, i=1, \ldots, M$. Перепишем уравнение (4) в виде (8), в котором линейный дифференциальный оператор $Q$ представлен выражением

$$
Q=w_{u}+\sum_{i=1}^{M} w_{u_{x_{i}}} \partial_{x_{i}}+\sum_{i_{1}, i_{2}=1}^{M} w_{u_{x_{i_{1}} x_{i_{2}}}} \partial_{x_{i_{1}} x_{i_{2}}}+\cdots .
$$

Важно, что функция $w$ удовлетворяет линейному уравнению (9) с $Q$ вида (47). Соотношение (10) также справедливо.

Семейство $M$-мерных редукций уравнения (4) можно построить с помощью следующей теоремы, аналогичной теореме 1.

ТЕОРема 2. Пусть функиия и является решением нелинейного $M$-мерного уравнения

$$
F(u, w)=0,
$$

где функиия $F(u, w)$ удовлетворяет уравнению

$$
F_{u u}+F_{w w} \frac{F_{u}^{2}}{F_{w}^{2}}-2 F_{u w} \frac{F_{u}}{F_{w}}=M(F, u, w),\left.\quad F_{w}\right|_{F=0} \neq 0,
$$

а $M$ - некоторая функиия от $F, u, w$, удовлетворяющая условию

$$
\left.M(F, u, w)\right|_{F=0}=0 .
$$


Тогда функиия $\psi$,

$$
\psi \equiv E(u)=u_{t}+w_{x_{1}}
$$

является решением линейного уравнения

$$
\left(F_{u}+F_{w} Q\right) \psi=0 .
$$

Если, кроме того, функиия $\psi$ удовлетворяет нулевым граничным условиям, тогда u - решение нелинейного уравнения (4).

ДокАзАтЕЛьство. Доказательство этой теоремы совершенно аналогично доказательству теоремы 1. Чтобы вывести нелинейное уравнение (4) из уравнения (48), продифференцируем уравнение (48) по $x_{i}$ и $t$ :

$$
\begin{aligned}
E_{x_{i}} & :=F_{u} u_{x_{i}}+F_{w} w_{x_{i}}=0, \quad i>1, \\
E_{t} & :=F_{u} u_{t}+F_{w} w_{t}=0 .
\end{aligned}
$$

Если $\left.F_{w=0}\right|_{F=}=\left.F_{u=0}\right|_{F=0}$, то уравнения $(53),(54)$ становятся тождествами. Предположим, что $\left.F_{w}\right|_{F=0} \neq 0$ (случай $\left.F_{u}\right|_{F=0} \neq 0$ может быть рассмотрен аналогичным образом). Тогда из уравнения (53) получим

$$
w_{x_{i}}=-\frac{F_{u}}{F_{w}} u_{x_{i}}, \quad i=1,2, \ldots
$$

Рассмотрим вторые производные уравнения (48) по произвольным парам $x_{i}, x_{j}$ и подставим выражение (55) для $w_{x_{i}}, i \geqslant 1$ :

$$
F_{u} u_{x_{i} x_{j}}+F_{w} w_{x_{i} x_{j}}+u_{x_{i}} u_{x_{j}}\left(F_{u u}+F_{w w} \frac{F_{u}^{2}}{F_{w}^{2}}-2 F_{u w} \frac{F_{u}}{F_{w}}\right)=0 .
$$

Если $F$ удовлетворяет нелинейному уравнению (49), тогда из уравнения (56) получим

$$
F_{u} u_{x_{i} x_{j}}+F_{w} w_{x_{i} x_{j}}=0 \Rightarrow w_{x_{i} x_{j}}=-\frac{F_{u}}{F_{w}} u_{x_{i} x_{j}} .
$$

По индукции для производной любого порядка $D=\prod_{i=1}^{M} \partial_{x_{i}}^{n_{i}}$, где $n_{i}-$ произвольные целые числа, получим соотношение

$$
F_{u} D u+F_{w} D w=0 .
$$

Следовательно, для дифференциального оператора $Q$ (47) имеем

$$
E_{Q}:=F_{u} Q u_{x_{1}}+F_{w} Q w_{x_{1}}=0 .
$$

Теперь рассмотрим следующую комбинацию уравнений (54), (59):

$$
E_{t}+E_{Q}=0,
$$

имеющую вид

$$
F_{u} E(u)+F_{w} E(w)=0 .
$$

В силу соотношения (10) можно переписать уравнение (61) в виде (52), где функция $\psi$ представлена выражением (51). Если, кроме того, $\psi$ удовлетворяет нулевым граничным условиям, то $\psi \equiv E(u) \equiv 0$, что эквивалентно уравнению (4). Теорема доказана. 
Теперь следует сделать несколько замечаний, аналогичных замечаниям раздела 2.

1. Область определения переменных $x_{i}, i=1, \ldots, M$, может быть как ограниченной, так и неограниченной.

2. Уравнение (49) эквивалентно уравнению (12) с точностью до переобозначений $\xi \leftrightarrow u$

3. Если функция $u$ удовлетворяет уравнениям (4) и (48), причем $\left.F_{w}\right|_{F=0} \neq 0$, то она удовлетворяет уравнению

$$
u_{t}-\frac{F_{u}}{F_{w}} u_{x_{1}}=0
$$

В частности, если функцию $F$ взять в виде $F=u+w$, то из уравнения $(28)$ получим

$$
u_{t}=u_{x_{1}} .
$$

Чтобы получить уравнение (62), нужно продифференцировать уравнение (48) по $x_{1}$, решить полученное уравнение относительно $w_{x_{1}}$ и подставить полученное $w_{x_{1}}$ в уравнение (4).

4. Поскольку дифференциальный оператор $Q(47)$ определяется функцией $w$ из уравнения (4), то нулевые граничные условия для функции $\psi(51)$ полностью определяются указанной функцией $w$ и не зависят от частного выбора функции $F(u, w)$.

ПримеР 2. В качестве примера рассмотрим нелинейное уравнение

$$
u_{t}+u_{x_{1} x_{2}}-\left(u^{3}\right)_{x_{1}}=0 .
$$

В этом случае

$$
w=u_{x_{2}}-u^{3} .
$$

Уравнение (49) имеет простое решение $(M=0)$

$$
F(u, w)=w-\gamma_{1} u-\gamma_{0},
$$

соответствующее редукции $u_{t}=-\gamma_{1} u_{x_{1}}$ в уравнении (4). При этом уравнение (48) принимает вид

$$
u_{x_{2}}=u^{3}+\gamma_{1} u+\gamma_{0} .
$$

Интегрируя ОДУ (67), получим

$$
\begin{gathered}
\frac{1}{\left(u_{1}-u_{2}\right)\left(u_{1}-u_{3}\right)} \ln \left(u-u_{1}\right)+\frac{1}{\left(u_{2}-u_{1}\right)\left(u_{2}-u_{3}\right)} \ln \left(u-u_{2}\right)+ \\
+\frac{1}{\left(u_{3}-u_{1}\right)\left(u_{3}-u_{2}\right)} \ln \left(u-u_{3}\right)=x_{2}+C\left(x_{1}, t\right),
\end{gathered}
$$

где $u_{1}, u_{2}$ и $u_{3}$ - корни полиномиального уравнения

$$
u^{3}+\gamma_{1} u+\gamma_{0}=0
$$

В частности, если $\gamma_{0}=0, \gamma_{1}=-1$, то, проинтегрировав уравнения (67), получим

$$
u^{2}=\frac{1}{1+\widetilde{C}\left(x_{1}, t\right) e^{2 x_{2}}} .
$$


Функция $C$ (или $\widetilde{C}$ ) не может быть произвольной, поскольку должна удовлетворять нулевому граничному условию для функции $\psi$,

$$
\psi=u_{t}+u_{x_{1} x_{2}}-\left(u^{3}\right)_{x_{1}}
$$

рассматриваемой в качестве решения линейного уравнения первого порядка (52) с $F_{u}=-\gamma_{1}, F_{w}=1$ и $Q=\partial_{x_{2}}-3 u^{2}$. Итак, существует единственное граничное условие в точке, например $x_{2}=0$ :

$$
\left.\psi\right|_{x_{2}=0}=0, \quad \text { при этом }\left.u\right|_{x_{2}=0}=\chi\left(x_{1}, t\right) .
$$

Выпишем уравнение $(62)$ с $F$ в виде $(66)$ в граничной точке $x_{2}=0$ :

$$
\chi_{t}+\gamma_{1} \chi_{x_{1}}=0
$$

Таким образом,

$$
\chi=A(\eta)
$$

где

$$
\eta=x_{1}-\gamma_{1} t
$$

Подставляя $\chi$ из уравнения (74) в уравнения (68) и (70) при $x_{2}=0$, замечаем, что функции $C$ и $\widetilde{C}$ должны зависеть от одной переменной $\eta$. Итак, $C$ и $\widetilde{C}$ можно считать произвольными функциями переменной $\eta$ в уравнениях (68) и (70), а граничное условие $(72)$ следует считать определением функции $A(\eta)$ через $C(\eta)$ или $\widetilde{C}(\eta)$ :

$$
\begin{aligned}
C(\eta)= & \frac{1}{\left(u_{1}-u_{2}\right)\left(u_{1}-u_{3}\right)} \ln \left(A(\eta)-u_{1}\right)+\frac{1}{\left(u_{2}-u_{2}\right)\left(u_{2}-u_{3}\right)} \ln \left(A(\eta)-u_{2}\right)+ \\
& +\frac{1}{\left(u_{3}-u_{1}\right)\left(u_{3}-u_{2}\right)} \ln \left(A(\eta)-u_{3}\right)
\end{aligned}
$$

и

$$
A^{2}(\eta)=\frac{1}{1+\widetilde{C}(\eta) e^{2 x_{2}}} .
$$

Если $\widetilde{C}$ - ограниченная положительная функция своего аргумента, то решение $(70)-$ ограниченное решение уравнения (64).

\section{4. О РЕШЕНИЯХ НЕЛИНЕЙНЫХ УРАВНЕНИЙ (12) И (49)}

Итак, мы свели $(M+1)$-мерное уравнение (4) к $M$-мерному уравнению (11) (если $w$ имеет вид (5)) или (48) (которое справедливо для любого $w$ ), где $F$ удовлетворяет двумерному уравнению второго порядка, уравнению (12) или (49) соответственно. Уравнения (11), (12) эквивалентны уравнениям (48), (49) с точностью до переобозначений $\xi \leftrightarrow u$. Совместим их в следующей паре уравнений:

$$
F(p, q)=0
$$

и

$$
F_{p p} F_{q}^{2}+F_{q q} F_{p}^{2}-2 F_{p q} F_{p} F_{q}=\mathcal{M}(F, p, q),
$$


симметричной по отношению к переменным $p$ и $q$. Переменную $p$ возьмем вместо $\xi$ или $u$, а переменную $q$ - вместо $w$. Кроме того, функция $\mathcal{M}$ должна удовлетворять условию

$$
\left.\mathcal{M}(F, p, q)\right|_{F(p, q)=0}=0 .
$$

Уравнение (79) можно считать условием совместности $(M+1)$-мерного уравнения (4) и $M$-мерного уравнения (78). В разделах 2 и 3 рассмотрены примеры частных решений нелинейного уравнения (4), связанные с простейшим решением уравнения (79). Однако это уравнение обладает богатым многообразием решений, параметризованных двумя произвольными функциями одной переменной, и заслуживает подробного изучения, которое не проводится в настоящей статье. Отметим только, что уравнение $(79)$ с $\mathcal{M}=0$ линеаризуется преобразованием Лежандра и его общее решение было найдено. Однако для такой функции $F$ уравнение $(78)$ всегда сводится к линейной зависимости между $p$ и $q$ (см. ниже пример 3$)$ и не представляет для нас интереса. Общий случай $\mathcal{M} \neq 0$ остается более обещающим.

Ниже рассмотрены только три частных решения уравнения (79) в виде вырожденной функции переменных $p$ и $q$

$$
F(p, q)=\sum_{i} f_{i}(p) g_{i}(q)
$$

ПримеР 3. Простейшее решение имеет вид

$$
F=\alpha_{0}+\alpha_{1} p+\alpha_{2} q
$$

где $\alpha_{i}, i=0,1,2,-$ произвольные постоянные параметры. Именно это решение используется в примере $1\left(\alpha_{0}=0, \alpha_{1}=\alpha_{2}=1\right)$ и примере $2\left(\alpha_{0}=-\gamma_{0}, \alpha_{1}=-\gamma_{1}\right.$, $\left.\alpha_{2}=1\right)$. В этом случае $\mathcal{M}=0$ в уравнении (79).

Отметим, что локальное линейное разложение функции $F(p, q)$ в окрестности фиксированной точки $\left(p_{0}, q_{0}\right)$ имеет вид (предполагается существование первых производных функции $F$ в этой точке)

$$
F(p, q) \approx F\left(p_{0}, q_{0}\right)+F_{p}\left(p_{0}, q_{0}\right)\left(p-p_{0}\right)+F_{q}\left(p_{0}, q_{0}\right)\left(q-q_{0}\right),
$$

что совпадает с выражением (82), если $\alpha_{0}=F\left(p_{0}, q_{0}\right)-F_{p}\left(p_{0}, q_{0}\right) p_{0}-F_{q}\left(p_{0}, q_{0}\right) q_{0}$, $\alpha_{1}=F_{p}\left(p_{0}, q_{0}\right), \alpha_{2}=F_{q}\left(p_{0}, q_{0}\right)$. Следовательно, функция (82) отвечает за локальную разрешимость уравнения (4).

ПримеР 4. В качестве следующего решения предлагается функция

$$
F=e^{\alpha p}+c_{2} e^{\beta p+c_{1} q}+c_{3} e^{\alpha c_{1} q /(\alpha-\beta)},
$$

где $\alpha, \beta, c_{i}, i=1,2,3,-$ произвольные параметры. В этом случае

$$
\begin{gathered}
\mathcal{M}=a_{1} F+a_{2} F^{2} \\
a_{1}=-\left(\frac{\alpha c_{1}}{\alpha-\beta}\left((\alpha-\beta) c_{2} e^{\beta p+c_{1} q}+\alpha c_{3} e^{\alpha c_{1} q /(\alpha-\beta)}\right)\right)^{2}, \\
a_{2}=\left(\alpha c_{1}\right)^{2}\left(c_{2} e^{\beta p+c_{1} q}+\frac{\alpha^{2} c_{3}}{(\alpha-\beta)^{2}} e^{\alpha c_{1} q /(\alpha-\beta)}\right) .
\end{gathered}
$$


ПримеР 5. Приведем еще один пример решения уравнения (79):

$$
F=e^{2 p}+2 e^{p+q c_{1} / 2} \sqrt{c_{2}} \sin \left(q c_{3}+c_{4}\right)+e^{q c_{1}} c_{2},
$$

где $c_{i}, i=1,2,3,4,-$ произвольные параметры. В этом случае

$$
\begin{aligned}
\mathcal{M}= & \left(a_{1}+a_{2} e^{p}\right) F+\left(a_{3}+a_{4} e^{p}\right) F^{2}, \\
a_{1}= & -4 e^{2 q c_{1}} c_{2}^{2}\left(c_{1} \cos \left(q c_{3}+c_{4}\right)-2 c_{3} \sin \left(q c_{3}+c_{4}\right)\right)^{2}, \\
a_{2}= & -2 e^{3 q c_{1} / 2} c_{2}^{3 / 2}\left(4 c_{1} c_{3} \cos \left(3\left(q c_{3}+c_{4}\right)\right)+\right. \\
& \left.+2\left(c_{1}^{2}+2 c_{3}^{2}+\left(c_{1}^{2}-4 c_{3}^{2}\right) \cos \left(2\left(q c_{3}+c_{4}\right)\right)\right) \sin \left(q c_{3}+c_{4}\right)\right), \\
a_{3}= & 4 e^{q c_{1}} c_{2}\left(c_{1} \cos \left(q c_{3}+c_{4}\right)-2 c_{3} \sin \left(q c_{3}+c_{4}\right)\right)^{2}, \\
a_{4}= & -2 e^{q c_{1} / 2} \sqrt{c_{2}}\left(4 c_{1} c_{3} \cos \left(q c_{3}+c_{4}\right)+\left(c_{1}^{2}-4 c_{3}^{2}\right) \sin \left(q c_{3}+c_{4}\right)\right) .
\end{aligned}
$$

\section{5. СПЕКТРАЛЬНОЕ УРАВНЕНИЕ, СВЯЗАННОЕ С НЕЛИНЕЙНЫМ УРАВНЕНИЕМ (4)}

Пространство решений можно расширить введением произвольных параметров (так называемых спектральных параметров) в функцию $F$, которые можно ввести с помощью функции $\mathcal{M}$ в правой части уравнения (79). Эти параметры появляются в решении (84) (параметры $\left.\alpha, \beta, c_{i}, i=1,2,3\right)$ и в решении (86) (параметры $c_{i}$, $i=1,2,3,4)$. Кроме того, произвольные функции одной переменной (обозначим их $\left.C_{i}(q), i=1,2\right)$ появляются в процессе интегрирования двумерного нелинейного уравнения второго порядка (79). Назовем векторным спектральным параметром $\lambda$ набор всех произвольных параметров и используем его следующим образом.

Вместо переменной $w$ (которая зависит от функции $u$ и ее производных по $x$ ) в списке аргументов функции $F$ будем использовать другую переменную $W\left(\lambda, C_{1}, C_{2}\right)$, связанную с $w$ интегральным выражением

$$
w\left(u, u_{x}, u_{x x}, \ldots\right)=\int D C_{1} D C_{2} \int d \Omega(\lambda) W\left(\lambda, C_{1}, C_{2}\right),
$$

где $\Omega$ - некоторая мера в пространстве векторного параметра $\lambda$, а $\int D C_{1} D C_{2}$ означает функциональное интегрирование по отношению к функциям $C_{i}, i=1,2$. Это уравнение следует рассматривать как уравнение для функции $u$, не зависящей от $\lambda$. Теперь мы должны заменить уравнения (11) и (48) соответственно уравнениями

$$
\begin{aligned}
& F\left(\xi, W\left(\lambda, C_{1}, C_{2}\right), \lambda\right)=0, \\
& F\left(u, W\left(\lambda, C_{1}, C_{2}\right), \lambda\right)=0 .
\end{aligned}
$$

Эти уравнения нужно решить относительно $W$. Применяя алгоритм, использованный в разделах 2 и 3 , выведем уравнения

$$
\begin{gathered}
F_{\xi} t E(u)+F_{W} E(W)=0, \\
F_{u} E(u)+F_{W} E(W)=0
\end{gathered}
$$

вместо уравнений (26) и (61) соответственно. Здесь

$$
E(W)=W_{t}+Q W_{x_{1}} .
$$


Разделим уравнения (91) и (92) на $F_{W}$ и применим оператор $\int D C_{1} D C_{2} \int d \Omega(\lambda)$. В силу соотношений (88) и (10) получим

$$
\begin{aligned}
& \left(\int D C_{1} D C_{2} \int d \Omega(\lambda) \frac{F_{\xi} t}{F_{W}}+Q\right) \psi=0, \\
& \left(\int D C_{1} D C_{2} \int d \Omega(\lambda) \frac{F_{u}}{F_{W}}+Q\right) \psi=0 .
\end{aligned}
$$

Заметим, что $Q$ и $\psi$ различны в уравнениях (94) и (95) (см. (7), (14) и (47), (51) соответственно). Теперь можно использовать нулевые граничные условия, о которых говорится в теоремах 1 и 2. Окончательно, решая уравнение (88) для функции $и$ с указанными граничными условиями, мы получим решение исходного $(M+1)$-мерного уравнения.

Вернемся теперь к уравнениям (91) и (92). Поскольку найденная функция $u$ является решением исходного уравнения в частных производных, первые слагаемые в уравнениях (91) и (92) исчезают. Следовательно, поскольку $F_{W} \neq 0$, получим линейное уравнение для спектральной функции $W(\lambda)$ :

$$
W_{t}\left(\lambda, C_{1}, C_{2}\right)+Q W_{x_{1}}\left(\lambda, C_{1}, C_{2}\right)=0 \text {. }
$$

Это уравнение можно считать спектральным для нелинейного уравнения (4). Подчеркнем, что, в отличие от МОЗР, у нас есть только одно спектральное уравнение, связанное с данным нелинейным уравнением. Следовательно, уравнение (4) не является условием совместности для некоторой переопределенной системы линейных уравнений.

5.1. О полноте пространства решений $(M+1)$-мерного уравнения (4). Богатство пространства решений $(M+1)$-мерного уравнения (4) общего вида определяется двумя наборами произвольных функций. Первый набор состоит из двух произвольных функций одной переменной, которые появляются в общем решении двумерного уравнения второго порядка (79) с фиксированной функцией $\mathcal{M}$. Функция $\mathcal{M}$ является произвольной функцией трех аргументов (ограниченной единственным условием (80)), которая, кроме того, может произвольным образом зависеть от спектрального параметра $\lambda$. Второй набор появляется в общем решении одного из уравнений $(11),(48)$ или (88), которые являются $M$-мерными (в общем случае) нелинейными уравнениями, поскольку $w-M$-мерное дифференциальное выражение от $u$. Таким образом, его общее решение зависит от произвольных функций $M$ переменных: $M-1$ переменных из набора $x$, дополненных переменной $t$. Однако эти функции удовлетворяют нулевым граничным условиям для функции $\psi=u_{t}+w_{x_{1}}$, о которых говорится в теоремах 1 и 2. Этот факт вносит дополнительные ограничения на указанные произвольные функции. Пока остается без ответа вопрос о возможности добиться полной интегрируемости нелинейного уравнения (4), комбинируя эти два набора произвольных функций вместе с произвольной функцией $\mathcal{M}$ и спектральным параметром. Эта проблема требует дальнейшего изучения. Роль функционального интегрирования в уравнении (88) также требует разъяснения. 


\section{6. РАЗРЕШИМОСТЬ УРАВНЕНИЙ (11) И (48)}

После построения функции $F(p, q)$ нужно решить $M$-мерное уравнение (11) или (48) на функцию $u$. Таким образом, возникает проблема интегрирования этих уравнений. В качестве простого примера рассмотрим уравнение (48) с $F(u, w)=$ $u+w:$

$$
u+w=0
$$

Это уравнение интегрируется, например, в следующих случаях.

6.1. Уравнение (97), интегрируемое с помощью МОЗР: уравнение коротких импульсов. Сначала рассмотрим случай уравнения (97), интегрируемого с помощью МОЗР. Например, пусть уравнение (97) имеет вид уравнения коротких импульсов [18], [19]

$$
u_{x_{2} x_{3}}+\left(u^{3}\right)_{x_{2} x_{2}}+u=0
$$

T. e.

$$
w=u_{x_{2} x_{3}}+\left(u^{3}\right)_{x_{2} x_{2}} .
$$

В этом случае уравнение (4) имеет вид

$$
u_{t}+\left(u_{x_{2} x_{3}}+\left(u^{3}\right)_{x_{2} x_{2}}\right)_{x_{1}}=0
$$

и может считаться дополнительным коммутирующим потоком уравнения коротких импульсов (98). Напомним, что не любые решения уравнения коротких импульсов удовлетворяют уравнению (100), а только удовлетворяющие нулевым граничным условиям для функции

$$
\psi=u_{t}+\left(u_{x_{2} x_{3}}+\left(u^{3}\right)_{x_{2} x_{2}}\right)_{x_{1}},
$$

как решения двумерного уравнения (52), в котором $F_{u}=F_{w}=1$ и дифференциальный оператор $Q$ имеет вид

$$
Q=6\left(u_{x_{2}}^{2}+u u_{x_{2} x_{2}}\right)+12 u u_{x_{2}} \partial_{x_{2}}+3 u^{2} \partial_{x_{2} x_{2}}+\partial_{x_{2} x_{3}} .
$$

6.2. Линеаризуемое уравнение (97). Рассмотрим линеаризуемое уравнение (97). Например, если $w=-\ln \left(u_{x_{3}}+u_{x_{2} x_{2}}-u_{x_{2}}^{2}\right)$, то уравнение (97) линеаризуется подстановкой $u=-\ln \varphi$, принимая вид

$$
\ln \left(-\varphi_{x_{3}}-\varphi_{x_{2} x_{2}}\right)=0 \quad \Rightarrow \quad \varphi_{x_{3}}+\varphi_{x_{2} x_{2}}+1=0
$$

Соответствующее уравнение (4) запишем в виде

$$
u_{t}-\ln _{x_{1}}\left(u_{x_{3}}+u_{x_{2} x_{2}}-u_{x_{2}}^{2}\right)=0
$$

При этом функция $\psi=u_{t}-\ln _{x_{1}}\left(u_{x_{3}}+u_{x_{2} x_{2}}-u_{x_{2}}^{2}\right)$ должна удовлетворять нулевым граничным условиям для уравнения (52) с $F_{u}=F_{w}=1$ и $Q=-\left(\partial_{x_{3}}+\partial_{x_{2} x_{2}}-\right.$ $\left.2 u_{x_{2}} \partial_{x_{2}}\right) /\left(u_{x_{3}}+u_{x_{2} x_{2}}-u_{x_{2}}^{2}\right)$. 
6.3. Уравнение (97), сводящееся к ОДУ. Рассмотрим случай, когда само уравнение (97) можно представить в виде (4):

$$
u+w \equiv u_{x_{1}}+w_{x_{2}}^{(1)}\left(u, u_{y_{1}}, u_{y_{1} y_{1}}, \ldots\right)=0
$$

с некоторой функцией $w^{(1)}$ от $u$ и ее производных по $x_{i}, i=2,3, \ldots, M$. Здесь мы используем наборы $y_{i}=\left\{x_{i+1}, \ldots, x_{M}\right\}, i=1,2, \ldots, M-1$, переменных $x_{i}$ и функции $w^{(i)}\left(u, u_{y_{i}}, u_{y_{i} y_{i}}, \ldots\right)$, зависящие от функции $u$ и ее производных по $x_{i+1}, x_{i+2}, \ldots, x_{M}$. Таким образом, уравнение (104) имеет решения, задаваемые уравнением вида (97):

$$
u+w^{(1)}=0 .
$$

В свою очередь, если уравнение (105) представимо в виде (4),

$$
u+w^{(1)} \equiv u_{x_{2}}+w_{x_{3}}^{(2)}\left(u, u_{y_{2}}, u_{y_{2} y_{2}}, \ldots\right)=0,
$$

то оно имеет решения, задаваемые уравнением вида (97):

$$
u+w^{(2)}=0,
$$

и т. д. Если этот процесс можно продолжить, то на $i$-м шаге получим уравнение

$$
u+w^{(i-1)} \equiv u_{x_{i}}+w_{x_{i+1}}^{(i)}\left(u, u_{y_{i}}, u_{y_{i} y_{i}}, \ldots\right)=0,
$$

обладающее решениями, задаваемыми уравнением вида (97):

$$
u+w^{(i)}=0 .
$$

На последнем шаге $y_{M-1}=x_{M}$, и мы приходим к ОДУ

$$
u+w^{(M-1)}\left(u, u_{x_{M}}, u_{x_{M} x_{M}}, \ldots\right)=0 .
$$

На каждом шаге необходимо обеспечить выполнение нулевых граничных условий для функции $\psi^{(i)}=u_{x_{i}}+w_{x_{i+1}}^{(i)}$ как решения линейного дифференциального уравнения

$$
\left(1+Q^{(i)}\right) \psi^{(i)}=0
$$

с дифференциальным оператором

$$
Q^{(i)}=w_{u}^{(i)}+\sum_{j=1}^{M} w_{u_{x_{j}}}^{(i)} \partial_{x_{j}}+\sum_{j_{1}, j_{2}=1}^{M} w_{u_{x_{j_{1}} x_{j_{2}}}}^{(i)} \partial_{x_{j_{1}} x_{j_{2}}}+\cdots
$$

Напомним, что само уравнение (104) сопровождается нулевыми граничными условиями для функции $\psi=u_{t}+w_{x_{1}}$ как решения уравнения $(1+Q) \psi=0$ с оператором $Q$, заданным выражением (47).

Простейшим уравнением, решения которого можно строить описанным выше способом, является уравнение

$$
u_{t}+\left(u_{x_{1}}-u+f_{x_{2}}(u)\right)_{x_{1}} \equiv u_{t}-u_{x_{1}}+u_{x_{1} x_{1}}+f_{x_{2} x_{1}}(u)=0
$$

где $f$ - произвольная функция от $u$. 


\section{7. ВЫВОдЫ}

Построен алгоритм, сводящий большой класс $(M+1)$-мерных нелинейных эволюционных уравнений, представимых в виде одномерного потока (4), к семейству $M$-мерных нелинейных уравнений (48) (или (11) для $w$ вида (5)). Совместность $(M+1)$-мерных и $M$-мерных уравнений приводит к двумерному уравнению второго порядка (79) для функции $F$. Важно то, что это уравнение справедливо для любого $(M+1)$-мерного уравнения вида $(4)$. Кроме того, в уравнение для $F$ можно ввести векторный спектральный параметр. Наличие такого параметра приводит к единственному линейному спектральному уравнению, связанному с исходным $(M+1)$-мерным уравнением. Однако второго спектрального уравнения, которое могло бы составить представление Лакса, не существует, в отличие от МОЗР. Разрешимость уравнения для $F$ - первый непростой шаг при построении частных решений, поскольку это уравнение нелинейное. Этот вопрос будет изучаться в отдельной статье. В настоящей статье приведено несколько частных решений этого уравнения (см. раздел 4).

Другой проблемой является разрешимость $M$-мерного уравнения $F=0$ относительно функции $u$, в которое входит функция $w$. Это уравнение следует изучать отдельно в каждом случае. В некоторых случаях можно свести $(M+1)$-мерное уравнение в частных производных к ОДУ, которое иногда допускает явные решения, как показано в приведенных выше примерах.

Очевидно существование большого многообразия нелинейных уравнений из описанного класса, имеющих физическое приложение. Можно обратиться к нелинейным уравнениям, рассмотренным в статье [17], которые также относятся к нашему случаю. В примере 1 рассмотрена деформация одного из этих уравнений.

Благодарности. Автор выражает благодарность А. Б. Шабату за важные замечания и Л. В. Богданову за полезное обсуждение. Работа финансировалась в рамках Программы поддержки ведущих научных школ (грант НШ-6170.2012.2).

\section{Список литературы}

[1] Дж. Уизем, Линейные и нелинейные волны, Мир, М., 1977.

[2] С. П. Царев, Докл. АН СССР, 282:3 (1985), 534-537.

[3] Б. А. Дубровин, С. П. Новиков, УМН, 44:6(270) (1989), 29-98.

[4] С. П. Царев, Изв. АН СССР. Сер. матем., 54:5 (1990), 1048-1068.

[5] E. V. Ferapontov, TMФ, 99:2 (1994), 257-262.

[6] E. Hopf, Commun. Pure Appl. Math., 3:3 (1950), 201-230.

[7] J. D. Cole, Quart. Appl. Math., 9 (1951), 225-236.

[8] F. Calogero, "Why are certain nonlinear PDEs both widely applicable and integrable?", What is Integrability?, ed. V. E. Zakharov, Springer, Berlin, 1991, 1-62.

[9] D. Li, Ya. G. Sinai, "Complex singularities of the Burgers system and renormalization group method", Current Developments in Mathematics, 2006, eds. D. Jerison, B. Mazur, T. Mrowka, W. Schmid, R. P. Stanley, S.-T. Yau, International Press, Somerville, MA, 2008, 181-210.

[10] D. Li, Ya. G. Sinai, J. Math. Phys., 51:1 (2010), 015205, 16 pp.

[11] C.S. Gardner, J. M. Green, M.D. Kruskal, R. M. Miura, Phys. Rev. Lett., 19:19 (1967), 1095-1097. 
[12] В. Е. Захаров, С.В. Манаков, С.П. Новиков, Л.П. Питаевский, Теория солитонов: Метод обратной задачи, Наука, М., 1980.

[13] M. J. Ablowitz, P. A. Clarkson, Solitons, Nonlinear Evolution Equations and Inverse Scattering, London Mathematical Society Lecture Note Series, 149, Cambridge Univ. Press, Cambridge, 1991.

[14] B. Konopel'chenko, Solitons in Multidimensions. Inverse Spectral Transform Method, World Sci., Singapore, 1993.

[15] В. Е. Захаров, А. Б. Шабат, Функи. анал. и его прил., 8:3 (1974), 43-53.

[16] В. Е. Захаров, А. Б. Шабат, Функи. анал. и его прил., 13:3 (1979), 13-22.

[17] A. I. Zenchuk, Particular solutions to multidimensional PDEs with KdV-type nonlinearity, arXiv: 1304.6864 .

[18] T. Schäfer, C. E. Wayne, Phys. D, 196:1-2 (2004), 90-105.

[19] A. Sakovich, S. Sakovich, J. Phys. Soc. Japan, 74:1 (2005), 239-241, arXiv: nlin/0409034.

Поступила в редакцию 19.09.2013 